\title{
Polarization of AGN in UV Spectral Range.
}

(Accepted for publication in Astrophysics \& Space Science)

\author{
Yu. N. Gnedin • M. Yu. Piotrovich • \\ T. M. Natsvlishvili
}

\begin{abstract}
We present the review of some new problems in cosmology and physics of stars in connection with future launching of WSO. We discuss three problems. UV observations of distant $z>6$ quasars allow to obtain information on the soft $<1 \mathrm{KeV} \mathrm{X}$-ray radiation of the accretion disk around a supermassive black hole because of its cosmological redshift. Really the region of X-ray radiation is insufficiently investigated because of high galactic absorption. In a result one will get important information on the reionization zone of the Universe. Astronomers from ESO revealed the effect of alignment of electric vectors of polarized QSOs. One of the probable mechanism of such alignment is the conversion of QSO radiation into low mass pseudoscalar particles (axions) in the extragalactic magnetic field. These boson like particles have been predicted by new SUSY particle physics theory. Since the probability of such conversion is increasing namely in UV spectral range one can expect the strong correlation between UV spectral energy distribution of QSO radiation and polarimetric data in the optical range. In the stellar physics one of the interesting problems is the origin of the X-ray sources with super Eddington luminosities. The results of UV observations of these X-ray sources will allow to find the origin of these sources as accreting intermediate mass black holes.
\end{abstract}

Keywords UV radiation, quasar, polarization;

Yu. N. Gnedin

M. Yu. Piotrovich

T. M. Natsvlishvili

Central Astronomical Observatory at Pulkovo, Saint-Petersburg, Russia.

\section{Introduction}

World Space Observatory - Ultraviolet (WSO-UV) presents the effective international project of a new space observatory intended for the investigation of the Universe in the UV range of the electromagnetic radiation spectrum (Shustov et al. 2009, 2011). The collection of scientific devices of this Observatory allows to provide solutions to the most important class of astronomical problems. It is very important that WSO-UV Observatory will realize the observations in the UV spectral region $(100 \div 320 \mathrm{~nm})$ inaccessible for ground based instruments. In our work we present an observational program which can be successful in the frame of this cosmic project.

The first program presents the quasar observations at the high cosmic distances $z=5 \div 6$. These QSOs have been discovered in the deep surveys (Fan et al. 2003; Jiang et al. 2007; Wang et al. 2008; Willot et al. 2007). UV radiation in the $100 \div 320 \mathrm{~nm}$ range corresponds to the rest frame radiation in the soft $h \nu \leq$ $0.5 \mathrm{KeV} \mathrm{X}$-ray spectral range. Namely this spectral region is inaccessible for the direct observations of the remote Universe because of the strong photoelectric absorption. The investigation of such remote QSOs is very important for understanding the origin of the reionization region that is located at the high cosmological distances $z \geq 6$.

The second program is including the investigations of physical processes at the intersection of cosmology and particle physics. We analyze the physical processes of the interaction of UV photons with intergalactic magnetic fields. One of the most curious recent astronomical discovery is the cosmological alignment of the electric vectors of polarization of distant QSOs (Hutsemekers et al. 2005; Sluse et al. 2005). One of the most probable physical mechanisms of this phenomenon is the process of the transformation of the photon into 
Table 1 Redshifts and continuum slopes.

\begin{tabular}{llr}
\hline Quasar & Redshift & Slope $_{U V}\left(\alpha_{\nu}\right)$ \\
\hline J0836+0054 & $5.810 \pm 0.003$ & $-0.62_{-0.06}^{+0.06}$ \\
$\mathrm{~J} 1030+0524$ & $6.309 \pm 0.009$ & $0.46_{-0.25}^{+0.18}$ \\
$\mathrm{~J} 1044-0125$ & $5.778 \pm 0.005$ & $-0.27_{-0.10}^{+0.09}$ \\
$\mathrm{~J} 1306+0356$ & $6.016 \pm 0.005$ & $0.50_{-0.14}^{+0.12}$ \\
$\mathrm{~J} 1411+1217$ & $5.927 \pm 0.004$ & $-0.21_{-0.19}^{+0.16}$ \\
\hline
\end{tabular}

axion in the magnetic field. Axion is predicted by the modern supersymmetry particle physics (SUSY). The probability of this process is increasing with photon energy and is especially high for UV photons. Therefore the observations of QSOs with high polarized radiation namely in UV range allow to obtain useful data about this physical process that is very important especially for modern particle physics.

The third section of our paper is devoted to the problem of the origin of X-ray sources with super Eddington luminosities. The solution of this problem can be obtained by the observation of these sources namely in UV spectral region. We expect that in result of such observations the intermediate mass black holes will be revealed. These objects are now considered as the most probable candidates of ultra-luminous X-ray sources.

\section{QSOs at High Cosmological Distances: the Prospects of UV Observations}

It is well known that the QSO radiation results from gas accretion on supermassive black holes (SMBHs). The $\mathrm{SMBH}$ is found in the central region of the active galactic nuclei (AGN). The typical values of SMBH masses are $10^{6} \div 10^{9} M_{\odot}$.

\subsection{The Study of the UV Slope}

The extremely high cosmological redshifts of a number of extragalactic objects have been recently revealed. For QSOs these redshifts are $z>6$, for galaxies they are $z>7$. The gamma-ray bursts have been recently detected at $z=8.2$. In deep surveys with the HST and ground based $8 \div 10 \mathrm{~m}$ telescopes the galaxies and quasars at very large cosmological distances $z \sim 7 \div 10$ have been detected ( $\operatorname{Li~2002)}$. The data derived from observations are including cosmological redshifts, power-law indices of the spectral energy distribution, masses of quasars, their bolometric luminosities and the Eddington ratio, i.e. the ratio of the bolometric luminosity to the value of the Eddington luminosity. The quasars at $z \sim 5 \div 6$ have their masses at the level of $M_{B H}=10^{9} \div 10^{10} M_{\odot}$ and the Eddington ratio: $l_{E}=L_{b o l} / L_{E d d} \sim 1$. Namely these objects are the prospective targets for observations at the cosmic observatory WSO-UV.

The spectral energy distribution in the rest frame of a typical quasar is dominated by a broad optical-UV hump normally identified with emission expected from an accretion disk surrounding a massive black hole. Another most important component of quasar radiation is $\mathrm{X}$-ray emission that is originated in the close vicinity of an accreting black hole. The effective power-law index is $\alpha \sim 0.5$ in the optical region and in the UV. For high redshift quasars index steepens further to $\alpha \sim 2$ in far UV and soft X-ray region and seems consistent with extrapolation to the soft X-ray excess detected in some quasars. Thus, the observations with WSO-UV will allow to get the important information on spectral energy distribution of high redshift quasars in soft $\mathrm{X}$-ray excess region.

Table 1 from Jiang et al. (2007) presents the redshifts and continuum slopes of high redshift QSOs. The continuum slope of quasar rest frame soft X-ray excess can be determined namely from UV observation in our system. The central BH masses and the bolometric luminosities are presented in Table 2 Jiang et al. 2007). The values of black hole masses are obtained from FWHM of CIV and MgII. $M_{B H}^{\prime}$ is estimated from the relation by Vestergaard \& Peterson (2006).

\subsection{The Study of QSO Magnetic Fields}

It is now commonly accepted that AGNs and QSOs possess the magnetized accretion disks. The magnetic coupling process is an effective mechanism for transferring energy and angular momentum from a rotating black hole to its surrounding accretion disk (Zhang. Lu \& Zhang 2005). Since the magnetic field on the horizon is brought and held by accretion disk matter, some relation must exist between the magnetic field strength and the BH mass and the bolometric luminosity (Silant'ev et al. 2009):

$$
\begin{aligned}
& B_{H}(G)=6.2 \times 10^{8}\left(\frac{M_{\odot}}{M_{B H}}\right)^{1 / 2}\left(\frac{L_{b o l}}{\varepsilon L_{E d d}}\right)^{1 / 2} \times \\
& \times \frac{1}{1+\sqrt{1-a_{*}^{2}}}
\end{aligned}
$$

This equation is valid for the equipartition condition at the BH horizon. In Eq.(11) $a_{*}$ is the Kerr parameter, the Eddington luminosity $L_{E d d}=1.3 \times$ $10^{38}\left(M_{B H} / M_{\odot}\right) \mathrm{erg} / \mathrm{s}$ and $\varepsilon$ is the radiation efficiency of the accretion process, i.e. $L_{b o l}=\varepsilon \dot{M} c^{2}$, where $\dot{M}$ is the accretion rate.

The results of our calculations of the $\mathrm{BH}$ magnetic field strengths at the reionization epoch $(z \geq 6)$ are 
Table 2 Central BH masses $\left(10^{9} M_{\odot}\right)$

\begin{tabular}{lccccc}
\hline Quasar & $L_{b o l}$ & $L_{b o l} / L_{E d d}$ & $M_{B H}(\mathrm{C} \mathrm{IV})$ & $M_{B H}(\mathrm{Mg}$ II $)$ & $M_{B H}^{\prime}(\mathrm{Mg}$ II $)$ \\
\hline J0836+0054 & 47.72 & 0.44 & $9.3 \pm 1.6$ & $\ldots$ & $\ldots$ \\
J1030+0524 & 47.37 & 0.50 & $3.6 \pm 0.9$ & $1.0 \pm 0.2$ & $2.1 \pm 0.4$ \\
J1044-0125 & 47.63 & 0.31 & $10.5 \pm 1.6$ & $\ldots$ & $\ldots$ \\
J1306+0356 & 47.40 & 0.61 & $3.2 \pm 0.6$ & $1.1 \pm 0.1$ & $2.2 \pm 0.3$ \\
J1411+1217 & 47.20 & 0.94 & $1.3 \pm 0.3$ & $0.6 \pm 0.1$ & $0.9 \pm 0.2$ \\
J1623+3112 & 47.33 & 1.11 & $\ldots$ & $1.5 \pm 0.3$ & $\ldots$ \\
\hline
\end{tabular}

Table 3 Magnetic field of QSOs at the epoch of reionization.

\begin{tabular}{lcccrrr}
\hline Quasar & $\mathrm{z}$ & $L_{\text {bol }} / L_{E d d}$ & $\begin{array}{c}a_{*}=0, \\
\varepsilon=0.057\end{array}$ & $\begin{array}{r}a_{*}=0.95, \\
\varepsilon=0.19\end{array}$ & $\begin{array}{r}a_{*}=0.998, \\
\varepsilon=0.32\end{array}$ & $\begin{array}{r}a_{*}=1.0, \\
\varepsilon=0.42\end{array}$ \\
\hline J0836+0054 & 5.810 & 0.44 & $9.0 \times 10^{3} \mathrm{G}$ & $7.5 \times 10^{3} \mathrm{G}$ & $7.15 \times 10^{3} \mathrm{G}$ & $6.6 \times 10^{3} \mathrm{G}$ \\
$\mathrm{J} 1030+0524$ & 6.309 & 0.50 & $1.5 \times 10^{4} \mathrm{G}$ & $1.22 \times 10^{4} \mathrm{G}$ & $1.16 \times 10^{4} \mathrm{G}$ & $1.0 \times 10^{4} \mathrm{G}$ \\
$\mathrm{J} 1044-0125$ & 5.778 & 0.31 & $7.1 \times 10^{3} \mathrm{G}$ & $6.0 \times 10^{3} \mathrm{G}$ & $5.7 \times 10^{3} \mathrm{G}$ & $5.3 \times 10^{3} \mathrm{G}$ \\
$\mathrm{J} 1306+0356$ & 6.016 & 0.61 & $1.8 \times 10^{4} \mathrm{G}$ & $1.5 \times 10^{4} \mathrm{G}$ & $1.43 \times 10^{4} \mathrm{G}$ & $1.4 \times 10^{4} \mathrm{G}$ \\
$\mathrm{J} 1411+1217$ & 5.927 & 0.94 & $3.5 \times 10^{4} \mathrm{G}$ & $2.93 \times 10^{4} \mathrm{G}$ & $2.8 \times 10^{4} \mathrm{G}$ & $2.7 \times 10^{4} \mathrm{G}$ \\
$\mathrm{J} 1623+3112$ & 6.247 & 1.11 & $3.5 \times 10^{4} \mathrm{G}$ & $2.93 \times 10^{4} \mathrm{G}$ & $2.8 \times 10^{4} \mathrm{G}$ & $2.7 \times 10^{4} \mathrm{G}$ \\
\hline
\end{tabular}

presented at Table 3 . The values of black hole masses $M_{B H}$ are taken from Table 2 (Jiang et al. 2007).

The magnetic field strengths and their topology in an accretion disk around the supermassive black hole can be determined by the UV polarimetric observations with use of Silant'ev and Gnedin method based onto the account of the Faraday rotation effect on the photon mean free path in the electron scattering process (Gnedin \& Silant'ev 1997; Silant'ev et al. 2009).

\subsection{The Study of Dust Emission in High Redshift QSOs}

Another effect which can be tested by WSO-UV is connected with the problem of existence of the dust and molecular torus around the SMBH in AGNs. In the local Universe the extremely UV and soft X-ray radiation are absorbed by this torus. The UV observations of high redshift QSOs allow to confirm absence or existence of the molecular torus at the early stages of the Universe evolution.

\section{Investigations of UV Background Radiation: Searching UV Photons Produced by Axion Decay and Photon Conversion into the Intergalactic Magnetic Field}

Axion is the most popular candidate in the dark matter. Though the axion as a weakly interacting particle there exist two most probable couplings of axions with the electromagnetic field (Fig.1): the decay of axion into two photons and the process of transformation
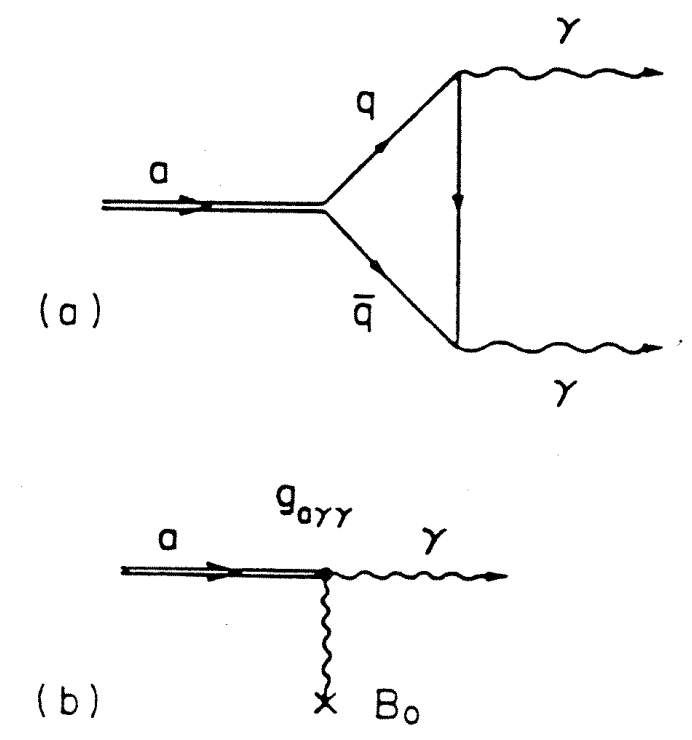

Fig. 1 (a) Two-photon coupling of the axion through a triangle anomaly; (b) Axion-photon transition in a magnetic field.

(conversion) of axion into photon and the reverse one of the photon conversion into axion.

Axions can decay into two photons. This $a \rightarrow 2 \gamma$ coupling arises due two different decay mechanisms: through axion-pion mixing and via electromagnetic (EM) anomaly of PQ symmetry in the standard QCD model. The axion decay time is (Ressel 1991):

$\tau_{a}(a \rightarrow 2 \gamma) \cong 6.8 \times 10^{24} \xi^{-2}\left(\frac{m_{a}}{1 e V}\right)^{-5} s$ 


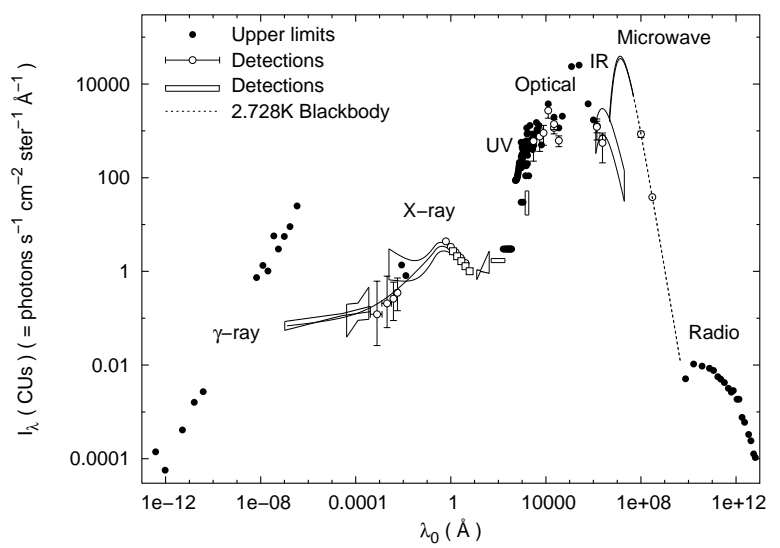

Fig. 2 A compilation of experimental measurements of the intensity of cosmic background radiation at all wavelengths.

where $\xi=|E / N-1.95| / 0.72, E$ and $N$ are the values of $\mathrm{EM}$ and color anomalies of PQ symmetry, respectively.

The free axion lifetime (2) is sufficiently large to allow for observations of decay of axions with mass much less than $1 \mathrm{eV}$. However, axion interaction with magnetic fields can provide photon production with energy comparable to the total axion energy (the Primakoff effect).

The probability of axion conversion into a photon and the inverse process of a photon conversion into an axion has the form (Gnedin 2002):

$P_{\|}(\gamma \leftrightarrow a)=\frac{1}{1+x^{2}} \sin ^{2} \frac{1}{2} B_{\perp} g_{a \gamma} L \sqrt{1+x^{2}}$

where $x=(\eta-1) \omega / 2 B g_{a \gamma}, \omega$ is the radiation frequency, $\eta$ is the dielectric permittivity of the medium in that the radiation is propagating, and $g_{a \gamma}$ is the constant of the interaction between axion and photon fields. The conversion process is very sensitive to the polarization state of the photon, since only a single polarization state, when the electric vector oscillates into the plane of the directions of the magnetic field and propagation of the photon, is subject to the conversion. Here $B_{\perp}=B \sin \theta$ where $\theta$ is the angle between the photon propagation and the magnetic field. A commonly accepted system of units in (3) is one for which $\hbar=c=1$. For pure vacuum solution of Eq.(3) transforms to

$P_{\|}(\gamma \leftrightarrow a)=\sin ^{2} \frac{1}{2} B_{\perp} g_{a \gamma} L \approx B_{\perp}^{2} L^{2} g_{a \gamma}^{2} / 4$

in the case of a weak effect of conversion.

The pseudoscalar axion like particles can contribute to observed spectral energy distribution of extragalactic background radiation. Fig.2 presents the spectral energy distribution of observed background radiation

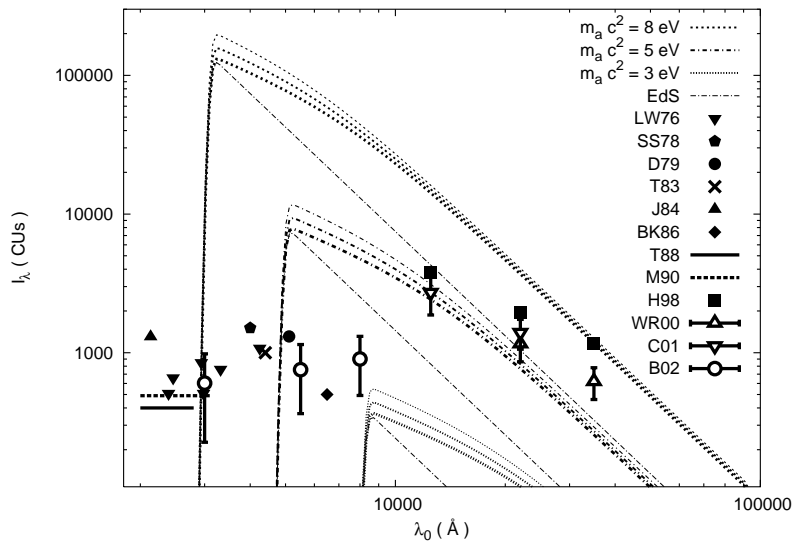

Fig. 3 The spectral intensity of the background radiation from decaying axions as a function of observed wavelength.

of the Universe (Overduin \& Wesson 2004). Now the researches are conducted for detection of the contribution of dark matter particles into the background photon spectrum. For example, the decay and annihilation of massive dark matter particles (WIMPs) can contribute in hard gamma-ray background radiation. In UV region one can expect an additional contribution to the background radiation of the Universe. We suggest that this contribution can be provided namely by the process of axion conversion into UV photon. The probability of this process increases with photon energy because the value of $\eta-1 \approx \omega_{P}^{2} / \omega^{2}$, where $\omega_{P}^{2}=4 \pi e^{2} N_{e} / m_{e}$ is the square of the plasma frequency, $N_{e}$ is the electron density of the intergalactic medium. Fig.3 (from Overduin \& Wesson (2004)) presents the comparison of the observed background radiation of the Universe with the expected contribution from the axion decay and transformation into intergalactic magnetic field (Piotrovich, Gnedin \& Natsvlishvili 2009).

\section{The Variability of Active Galactic Nuclei in the UV Spectral Region.}

Many AGNs show variability of their radiation. As a rule, UV variability scale is shorter that optical one (Lyuty 2005). It allows to investigate the wellknown mechanism of the magnetic reconnection and annihilation of the magnetic force lines in the nearest region of supermassive black holes. The characteristic time of this process is determined as $\Delta t=$ $R_{\lambda} / V_{\text {rec }}$, where $R_{\lambda}$ is the scale of an accretion disk that corresponds to the radiation wavelength $\lambda$ and $V_{\text {rec }} \sim V_{A}$ is the rate of reconnection process $\left(V_{A}\right.$ is the Alfven velocity). The scale of the standard accre- 
tion disk (Shakura \& Sunvaev 1973) was calculated by Poindexter, Morgan \& Kochanek (2007):

$R_{\lambda}=0.97 \times 10^{10} \lambda_{\text {rest }}^{4 / 3}\left(\frac{M_{B H}}{M_{\odot}}\right)^{2 / 3}\left(\frac{L_{b o l}}{\varepsilon L_{E d d}}\right)^{1 / 3}$

where $M_{B H}$ and $L_{b o l}$ are the mass and bolometric luminosity of a supermassive black hole, $\varepsilon$ is the coefficient of radiative efficiency of accreting matter and $L_{E d d}=1.3 \times 10^{38}\left(M_{B H} / M_{\odot}\right) \mathrm{erg} / \mathrm{s}$ is the Eddington luminosity, $\lambda_{\text {rest }}$ is the rest frame wavelength in $\mu \mathrm{m}$.

The relation between the typical variability scales in $\mathrm{UV}$ and visual region is

$\frac{\Delta t(U V)}{\Delta t(O p t)}=\left[\frac{\lambda_{\text {rest }}(U V)}{\lambda_{\text {rest }}(O p t)}\right]^{4 / 3} \frac{V_{\text {rec }}(O p t)}{V_{\text {rec }}(U V)}$

If we suggest that the reconnection velocity is proportional to the Alfven velocity and we use the standard model of an accretion disk, we obtain the reconnection time is proportional to $\lambda^{2}$.

Eq.(6) means that one can derive the magnetic field strength and its topology in the accretion disk in the case of simultaneous measurement of characteristic variability time in the UV and optical spectral regions, because the Alfven velocity is dependent essentially on the magnetic field.

\section{X-ray Sources with Supereddington Luminosity}

Recently a number of X-ray sources with extremal supereddington luminosity has been discovered (Watson et al. 2010). This fact allows to consider these sources as black holes of intermediate masses. In this case it is easy to explain the extremal luminosity of these objects.

The essential element of physical properties of these sources is high ratio of X-ray flux to optical radiation $F_{x} / F_{\text {opt }} \sim 150 \div 250$. This situation does not allow to make optical identification of these sources. However in the UV spectral region the situation is more optimistic because the ratio $F_{x} / F_{U V} \sim 10$ if we use the standard model of an accretion disk. This fact allows to expect the more favorable situation for identification of these objects in UV spectral range. For intermediate mass black holes the ratio of $F_{x} / F_{U V} \geq 1$, that is the decisive fact in the problem of identification of ultra luminous X-ray sources. Also another important task is to derive their variability scale and comparison of this scale with corresponding variability scales in X-ray and optical regions. In a result one should expect obtaining new important information on the origin of UV XRS.

\section{Conclusions}

Mentioned here tasks do not exhaust all possibilities of the remarkable WSO-UV space observatory. One can expect that this observatory provides the remarkable breakthrough in the investigation of all of the Universe and separate astronomical objects. One can expect the interesting results in new regions of close intersection of astronomy with fundamental particle physics. Many astrophysical objects can be considered as the particle physics laboratories. The origin of dark matter and dark energy is the problem which is very important for astronomy and fundamental physics. The solution of this problem may therefore lie in the existence of some new form of non-baryonic matter, such as, for example, axions.

The launching of WSO-UV Observatory will make essential contributions to solution of these problems.

\section{Acknowledgements}

This research was supported by the program of Prezidium of RAS "Origin and Evolution of Stars and Galaxies", the program of the Department of Physical Sciences of RAS "Extended Objects in the Universe", by the Federal Program "Scientific and Science-Teaching Staff of Innovation Russia" (GK 02.740.11.0246) and by the grant from President of the Russian Federation "The Basic Scientific Schools" NSh3645.2010.2. 


\section{References}

Fan X. et al., AJ, 125, 1649 (2003)

Gnedin Yu.N., Silant'ev N.A., Astrophys.Sp.Phys.Rev., 10, 1 (1997)

Gnedin Yu.N., Int.J.Modern.Phys., 17, 4251 (2002)

Hutsemekers D. et al., A\&A, 441, 915 (2005)

Jiang L. et al., AJ, 134, 1150 (2007)

Li L.X., A\&A, 392, 469 (2002)

Lyuty V.M., Astronomy Letters, 31, No.10, 645 (2005)

Ma R.-Y., Yang F., Wang D.X., ApJ, 671, 1981 (2007)

Overduin J.M., Wesson P.S., Phys.Rep., 402, 267 (2004)

Piotrovich M.Yu., Gnedin Yu.N., Natsvlishvili T.M., Astrofizika, 52, No.3, 412 (2009)

Poindexter S., Morgan N., Kochanek C.S., arXiv:0707.0003 (2007)

Ressel M.T., Phys.Rev., D44, 3001 (1991)

Shakura N.I., Sunyaev R.A., A\&A, 24, 337 (1973)

Shustov B. et al., Astrophys. Spase Sci., 320, 187 (2009)

Shustov B. et al., Astrophys. Spase Sci., this issue (2011)

Silant'ev N.A., Piotrovich M.Yu., Gnedin Yu.N., Natsvlishvili T.M., A\&A, 507, 171 (2009)

Sluse D. et al., A\&A, 443, 757 (2005)

Vestergaard M., Peterson B.M., ApJ, 641, 689 (2006)

Wang R. et al., arXiv:0806.3028 (2008)

Watson D.J., Gladstone J.C., Roberts T.P., Fabian A.C., arXiv:1011.0398 (2010)

Willot C.J. et al., AJ, 134, 2435 (2007)

Zhang D.W., Lu Y., Zhang S.N., Chin.J.Astron.Astrophys., 5, $347(2005)$

This manuscript was prepared with the AAS LATEX macros v5.2. 